\title{
A comparative study on intraocular pressure under various anesthetics in cynomolgus monkeys (Macaca fascicularis)
}

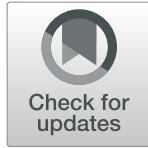

\author{
Hong-Soo Lee ${ }^{1 \dagger}$, Da-Hee Kim², Sung-Hwan Kim², Min-Sung Kang ${ }^{2}$ and Han Na Suh²
}

\begin{abstract}
Background: Nonhuman primates (NHPs) are superior model for ocular research due to its morphological and physiological similarities with humans. Thus, the effect of four different anesthetic combinations [ketamine (10 mg/ $\mathrm{kg})$, ketamine + xylazine $(7+0.6 \mathrm{mg} / \mathrm{kg})$, zoletil $(4 \mathrm{mg} / \mathrm{kg})$, and zoletil + xylazine $(4+0.2 \mathrm{mg} / \mathrm{kg})]$ on intraocular pressure (IOP) was determined in cynomolgus monkeys.

Results: The administration of ketamine + xylazine or zoletil + xylazine resulted in lower IOP compared to ketamine or zoletil alone. Moreover, the IOP in male monkeys was higher than in females. The difference between the right and left eye was not found.

Conclusions: Anesthetics affected the IOP, and gender differences should be considered when measuring the IOP of nonhuman primates (NHPs).
\end{abstract}

Keywords: Anesthetics, Cynomolgus monkey, Intraocular pressure, TonoVet ${ }^{\mathrm{TM}}$

\section{Background}

Preclinical study is a step to examine the drug feasibility and collect the drug safety data prior to clinical trial. Generally, regulatory guidelines indicate the use of rodent (mouse and rat) and/or non-rodent [nonhuman primates (NHP), porcine, canine] in preclinical study. To select the right species, pharmacological relevance based on literature, in silico, in vitro, ex vivo, and in vivo data should be considered [1, 2]. The canine is a proper testing species for new chemical entities. On the other hand, biotherapeutics such as monoclonal antibody, antisense oligonucleotide, or recombinant protein have potent pharmacological activity in NHPs [3, 4]. Porcine is the best model for dermal toxicity due to the histological and functional similarity of its skin to humans.

\footnotetext{
* Correspondence: hanna.suh@kitox.re.kr

${ }^{\dagger} \mathrm{H}$ ong-Soo Lee and Han Na Suh contributed equally to this work.

${ }^{2}$ Korea Institute of Toxicology, 30 Haekhak 1-gil, Jeongeup, Jeollabuk-do, South Korea

Full list of author information is available at the end of the article
}

Thus, minimum and proper NHP application is required in preclinical safety or efficacy study.

The intraocular pressure (IOP) is the pressure within the eye, which is based on the production and drainage of aqueous humor. IOP can be affected by various factors such as obesity [5], water consumption [6], stress [7], circadian rhythm [8], reproductive cycle [9], systemic blood pressure [10], and anesthesia [11]. In addition, ocular toxicity may be associated with IOP changes during the preclinical study. NHPs are superior model for ocular research due to its morphological and physiological similarities with humans $[9,12]$. All drugs which are clinical prescribed against glaucoma have been found to reduce the IOP in NHPs. The development of novel glaucoma medication is widely ongoing, utilizing NHPs $[13,14]$. Thus, an accurate, reproducible, and convenient IOP assessment for NHP is essential in preclinical studies.

To acquire the accurate IOP of NHPs, IOP is measured under anesthesia. Ketamine, a NMDA receptor antagonist, is commonly used as an anesthetic agents 
in NHPs [15]. Combination of ketamine with other dissociative anesthetics attenuates the reflex during anesthesia [16]. Zoletil is a veterinary anesthetic combination of tiletamine hydrochloride and zolazepam hydrochloride. Tiletamine is a NMDA receptor antagonist and zolazepam is an enhancer of GABA action having sedative, anxiolytic, and skeletal muscle relaxant effect [17]. Xylazine, an adrenergic receptor $\alpha 2$ agonist, is used for sedation, anesthesia, muscle relaxation, and analgesia in animals. Here we have investigated the effect of different combinations of anesthetics on the IOP of NHPs using TonoVet ${ }^{\mathrm{Tm}}$. This study may serve as a foundation of the IOP baseline when measuring the IOP in NHPs.

\section{Results}

Effect of four different combination of anesthetics on the IOP of cynomolgus monkeys

To determine the effect of anesthetics on IOP, we chose four different anesthetic combinations; ketamine, ketamine + xylazine, zoletil, or zoletil + xylazine. During the IOP measurement in NHPs, ketamine was used for sedation [18]. The animals under ketamine showed the steady IOP $(20.65 \pm 6.34$ to $19.35 \pm 9.00 \mathrm{mmHg})$, supporting ketamine as the suitable anesthetic during IOP measurement. However, ketamine + xylazine showed decreased IOP $(14.7 \pm 3.73$ to $11.6 \pm 3.00 \mathrm{mmHg})$. When we compared the IOP at $5 \mathrm{~min}$, ketamine + xylazine showed the lower IOP than ketamine alone. Zoletil is a more potent anesthetic regulating both NMDA receptor and GABA. We found that zoletil slightly decreased the IOP $(18.9 \pm 4.06$ to $15.68 \pm 3.57 \mathrm{mmHg})$, and zoletil + xylazine had similar decreased pattern $(14.78 \pm 4.08$ to $11.6 \pm 3.67 \mathrm{mmHg}$ ) (Fig. 1). Above all, ketamine had steady IOP, and adding xylazine decreased the IOP.

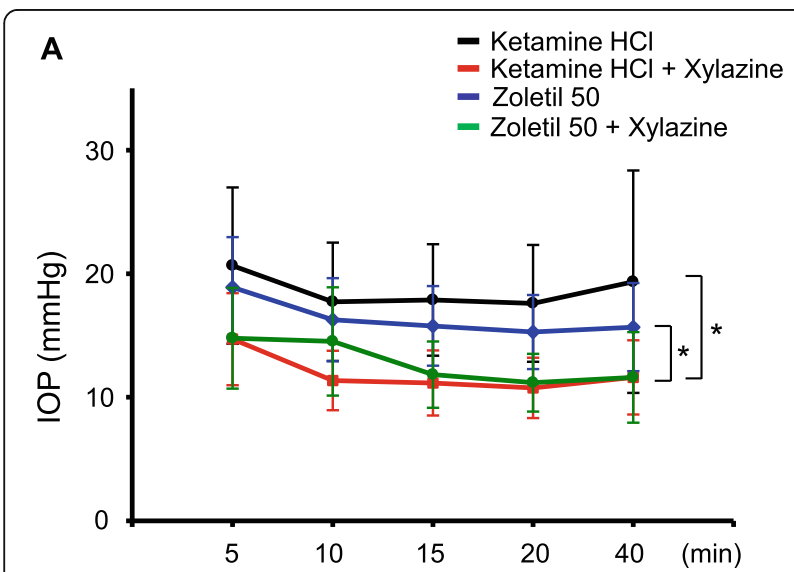

Fig. 1 Effect of anesthetics on the IOP in cynomolgus monkeys. (A) IOP was measured (5, 10, 15, 20, and 40 min) after anesthetization. Xylazine-treated animals showed significantly lower IOP compared to ketamine or zoletil alone-treated animals. ${ }^{*} p<0.05 . \mathrm{n}=20$ in each group (both eyes of five male and five female monkeys)

\section{Comparison of IOP between two genders and right/left eyes in cynomolgus monkeys}

To determine whether the IOP is affected by gender, we compared the IOP of female and male monkeys. We found that the male had higher IOP (from 22.5 \pm 3.30 to $27 \pm 7.29 \mathrm{mmHg}$ ) than the female (from $15.2 \pm 2.64$ to $15.3 \pm 1.75 \mathrm{mmHg}$ ) in ketamine. Other anesthetics were not different between genders (Fig. 2).

To examine whether the IOP had variations in both eyes, we analyzed the IOP of the right and left eye. The right and left eye had similar IOP values regardless of the anesthetic types (Fig. 3).

\section{Discussion}

In this study, we examined the IOP to compare the effects of four different anesthetics in male and female Vietnamese cynomolgus monkeys using rebound tonometer, TonoVet ${ }^{\mathrm{TM}}$. As cynomolgus monkeys can be a representative ocular disease model and are highly suitable for drug screening for glaucoma [12], we have utilized this strain for IOP measurement. TonoVet ${ }^{\mathrm{TM}}$ was validated as an accurate equipment for IOP measurement due to the high correlation with the actual eye pressure [18]. Another study found that the IOP values have greater variety when using TonoVet ${ }^{\mathrm{Tm}}$ compared to fixed tonometer [19]. Thus, technical consistency and proper position of TonoVet ${ }^{\mathrm{TM}}$ are essential to get reproducible IOP.

To acquire the representative IOP, NHPs were under general anesthesia during the procedure. It was reported that ketamine increases the IOP in cats, dogs, and rabbits. Ketamine with diazepam significantly elevates the IOP in dogs [13]. On the other hand, midazolam decreases the IOP in humans [20]. The median IOP was measured to be 10 to $25 \mathrm{mmHg}$ in most animals [21, 22]. We found that all measured IOP was in normal range, regardless of anesthetics used. The $\alpha 2$-adrenoceptor agonist, xylazine, decreased the IOP by reducing aqueous flow [23]. Of note, topical or systemic administration of atropine sulfate increases canine IOP [24]. As atropine is an anticholinergic drug, the differential effect of atropine on combined anesthetics is possible. Lions anesthetized with ketamine showed similar IOP regardless of atropine [25]. The possible interference or synergistic effect of combination of various anesthetics is need to be explored. As anesthetics affect the IOP, it should be noted that the basal IOP depends on the anesthetics when a researcher performs an ophthalmologic procedure.

Gender is another factor associated with the IOP in humans and lions $[25,26]$. We found that ketaminetreated male had higher IOP than female monkeys. Other anesthetic did not show the difference in IOP between the two genders. The differences of IOP based on gender might be related to sexual hormone or anesthetic sensitivity. Here, we demonstrated that xylazine decreased 

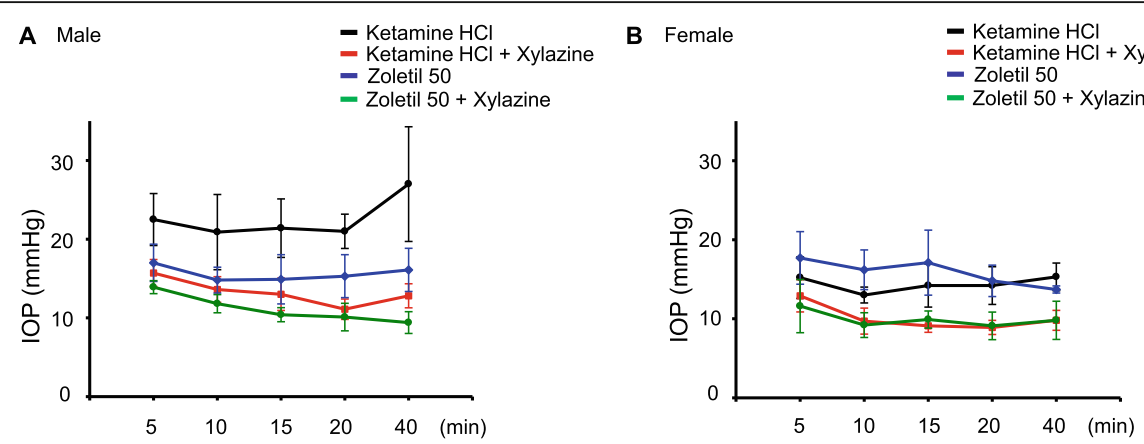

Fig. 2 A comparative study of IOP in male and female cynomolgus monkeys. (A-B) IOP was measured (5, 10, 15, 20, and 40 min) after anesthetization. The IOP in male (A) was higher than in female (B). ${ }^{*} p<0.05 . n=10$ in each group (both eyes of five male or five female monkeys)

the IOP significantly after administration, regardless of ketamine or zoletil combination. This study provides the baseline IOP of cynomolgus monkeys under various anesthetics, which might be helpful for scientists and pharmaceutical companies interested in glaucoma and eyes.

\section{Conclusions}

Anesthetics affected the IOP, and gender differences should be considered when measuring the IOP of nonhuman primates (NHPs).

\section{Methods}

\section{Animals, Husbandry, and Feeding}

Ten cynomolgus monkeys, (five males and five females; Macaca fascicularis), aged 2-3 year and weight 2.89-
$3.17 \mathrm{~kg}$, supplied from Nafo Vanny (Dong Nai, Vietnam). Monkeys were housed individually in a stainless cage $(540 \mathrm{~mm} \times 760 \mathrm{~mm} \times 850 \mathrm{~mm})$ with enrichments. Room temperature and humidity regulated $\left(20 \sim 29{ }^{\circ} \mathrm{C} ; 30 \sim\right.$ $70 \%)$. Fluorescent lighting 300-700 lx and air changes 10-20 times/hour maintained. Water was provided ad libitum and food was provided at the rate of $3 \%$ of the body weight per day. Fruits were provided twice a week. All the animal experiments were conducted under Institutional Animal Care and Use Committee guideline of the Korea Institute of Toxicology (IACUC KIT 1506 - 0158).

\section{Intraocular pressure measurements}

Any ophthalmic abnormalities were not detected during this procedure, as confirmed by slit-lamp biomicroscopy and funduscopy. The animals were pre-medicated with
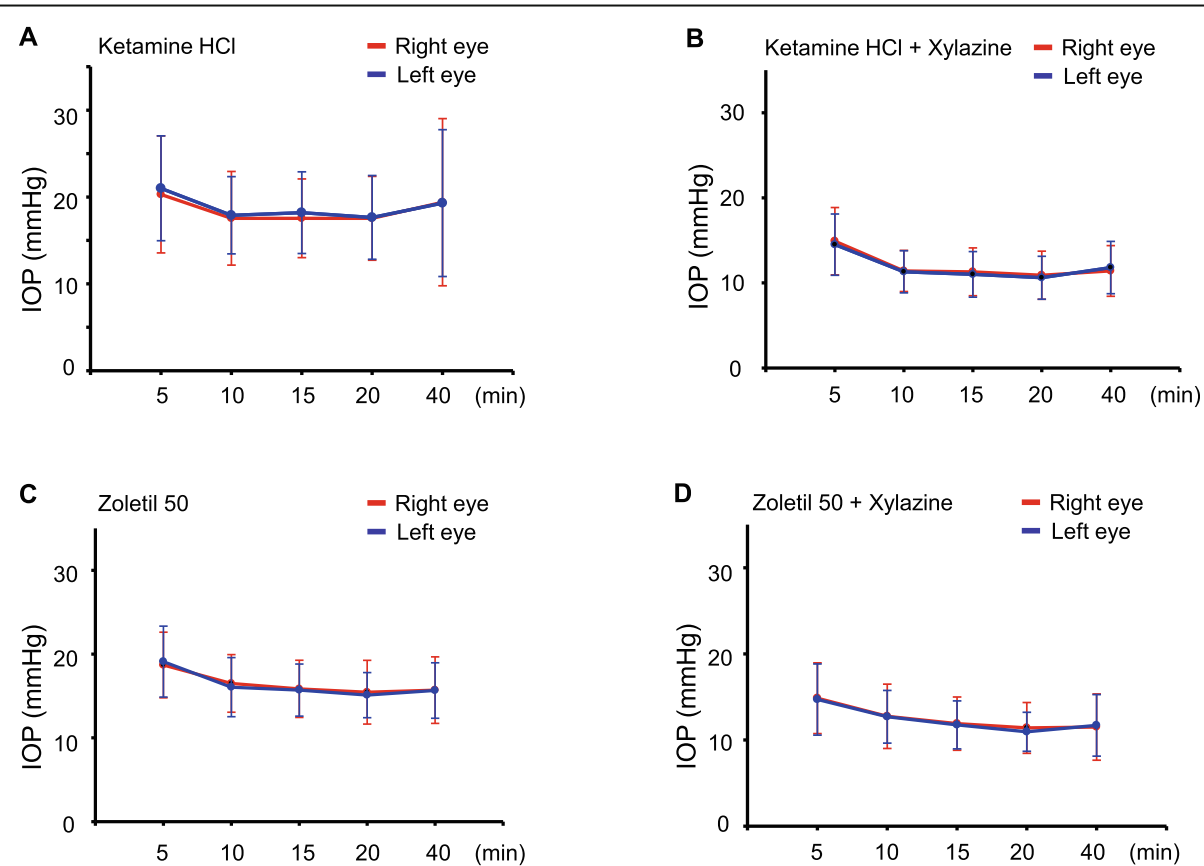

Fig. 3 A comparative study of IOP in right and left eyes. (A-D) IOP was measured (5, 10, 15, 20, and 40 min) after anesthetization. There was no difference in the IOP of right and left eyes. ${ }^{*} p<0.05 . n=10$ in each group (right or left eyes in both genders) 
atropine sulfate $(0.04 \mathrm{mg} / \mathrm{kg}, \mathrm{SC})$, followed by ketamine $(10 \mathrm{mg} / \mathrm{kg}$, IM), ketamine + xylazine $(7 \mathrm{mg} / \mathrm{kg}+0.6 \mathrm{mg} /$ $\mathrm{kg}$, IM), zoletil (4 $\mathrm{mg} / \mathrm{kg}$, IM), or zoletil + xylazine $(4 \mathrm{mg} / \mathrm{kg}+0.2 \mathrm{mg} / \mathrm{kg}$, IM). Then, IOP was measured in each eyes at $5,10,15,20$, and $40 \mathrm{~min}$ after anesthetization. IOP was measured bilaterally using the rebound tonometer (TonoVet ${ }^{\mathrm{Ts}}$, Vantaa, Finland), following the manufacturer's protocol. The right eye was measured first. TonoVet ${ }^{\mathrm{Tw}}$ software was programmed for six measurements (consecutive central corneal touches) and displayed the average of six values. All measurements were conducted in the same room between $6 \mathrm{pm}$ and 8 $\mathrm{pm}$ to minimize circadian variation.

\section{Statistical analysis}

The data were analyzed using the statistical package SPSS (SPSS Inc., IL, USA) for Windows version 12.0. Two-way ANOVA with repeated measures were used, followed by Bonferroni's test to compare the average IOP values of both eyes among groups. For IOP comparison between the right and left eye, Student's T-test was used. Levene's test was applied for the homogeneity of variance in both genders. The data are presented as the mean \pm standard deviation. The differences are considered significant when $p<0.05$.

\section{Abbreviations}

NHP: Nonhuman primates; IOP: Intraocular pressure

\section{Acknowledgements}

Not applicable.

\section{Authors' contributions}

H Lee, D Kim, and M Kang performed the experiments. H Lee and HN Suh analyzed and interpreted the results. HN Suh wrote the manuscript and prepared the figures. S Kim revised manuscript. All authors approved the final version of the manuscript.

\section{Funding}

This work was supported by the Korea Institute of Toxicology (KK-2109-01).

Availability of data and materials

All data are included in Results.

\section{Declarations}

\section{Competing interests}

All authors have no potential conflicts of interest.

\section{Author details}

${ }^{1}$ Biotoxtech, 53 Yeongudanji-ro, Ochang-eup, Chungcheongbuk-do, South Korea. ${ }^{2}$ Korea Institute of Toxicology, 30 Haekhak 1-gil, Jeongeup, Jeollabuk-do, South Korea.

Received: 7 April 2021 Accepted: 7 June 2021

Published online: 22 June 2021

\section{References}

1. Bussiere JL, Martin P, Horner M, Couch J, Flaherty M, Andrews L, et al. Alternative strategies for toxicity testing of species-specific biopharmaceuticals. Int J Toxicol. 2009;28(3):230-53.
2. Bussiere JL. Species selection considerations for preclinical toxicology studies for biotherapeutics. Expert Opin Drug Metab Toxicol. 2008;4(7):871-7.

3. Buckley LA, Chapman K, Burns-Naas LA, Todd MD, Martin PL, Lansita JA. Considerations regarding nonhuman primate use in safety assessment of biopharmaceuticals. Int J Toxicol. 2011;30(5):583-90.

4. Chapman K, Pullen N, Coney L, Dempster M, Andrews L, Bajramovic J, et al. Preclinical development of monoclonal antibodies: considerations for the use of non-human primates. MAbs. 2009;1 (5):505-16.

5. Mori K, Ando F, Nomura H, Sato Y, Shimokata H. Relationship between intraocular pressure and obesity in Japan. Int J Epidemiol. 2000;29(4):661-6.

6. Poon YC, Teng MC, Lin PW, Tsai JC, Lai IC. Intraocular pressure fluctuation after water drinking test in primary angle-closure glaucoma and primary open-angle glaucoma. Indian J Ophthalmol. 2016;64(12):919-23.

7. Shily BG. Psychophysiological stress, elevated intraocular pressure, and acute closed-angle glaucoma. Am J Optom Physiol Opt. 1987:64(11):866-70.

8. Liu JH. Circadian rhythm of intraocular pressure. J Glaucoma. 1998;7(2):141-7.

9. Ofri R, Horowitz IH, Raz D, Shvartsman E, Kass PH. Intraocular pressure and tear production in five herbivorous wildlife species. Vet Rec. 2002;151(9):265-8.

10. Klein BE, Klein R, Knudtson MD. Intraocular pressure and systemic blood pressure: longitudinal perspective: the Beaver Dam Eye Study. Br J Ophthalmol. 2005;89(3):284-7.

11. Jasien JV, Girkin CA, Downs JC. Effect of Anesthesia on Intraocular Pressure Measured With Continuous Wireless Telemetry in Nonhuman Primates. Invest Ophthalmol Vis Sci. 2019;60(12):3830-4.

12. Raghunathan V, Eaton JS, Christian BJ, Morgan JT, Ver Hoeve JN, Yang CC, et al. Biomechanical, ultrastructural, and electrophysiological characterization of the non-human primate experimental glaucoma model. Sci Rep. 2017; 7(1):14329.

13. Hofmeister EH, Mosunic CB, Torres BT, Ralph AG, Moore PA, Read MR. Effects of ketamine, diazepam, and their combination on intraocular pressures in clinically normal dogs. Am J Vet Res. 2006;67(7):1136-9.

14. Liu G, Zeng T, Yu W, Yan N, Wang H, Cai SP, et al. Characterization of intraocular pressure responses of the Tibetan monkey (Macaca thibetana). Mol Vis. 2011;17:1405-13.

15. Lee VK, Flynt KS, Haag LM, Taylor DK. Comparison of the effects of ketamine, ketamine-medetomidine, and ketamine-midazolam on physiologic parameters and anesthesia-induced stress in rhesus (Macaca mulatta) and cynomolgus (Macaca fascicularis) macaques. J Am Assoc Lab Anim Sci. 2010;49(1):57-63.

16. Sun FJ, Wright DE, Pinson DM. Comparison of ketamine versus combination of ketamine and medetomidine in injectable anesthetic protocols: chemical immobilization in macaques and tissue reaction in rats. Contemp Top Lab Anim Sci. 2003;42(4):32-7.

17. Lee Jl, Hong SH, Lee SJ, Kim YS, Kim MC. Immobilization with ketamine $\mathrm{HCl}$ and tiletamine-zolazepam in cynomolgus monkeys. J Vet Sci. 2003; 4(2):187-91.

18. Yu W, Cao G, Qiu J, Liu X, Ma J, Li N, et al. Evaluation of monkey intraocular pressure by rebound tonometer. Mol Vis. 2009:15:2196-201.

19. Kontiola Al. A new induction-based impact method for measuring intraocular pressure. Acta Ophthalmol Scand. 2000;78(2):142-5.

20. Fragen RJ, Hauch T. The effect of midazolam maleate and diazepam on intraocular pressure in adults. Arzneimittelforschung. 1981;31(12a):2273-5.

21. Peche N, Eule JC. Intraocular pressure measurements in cattle, sheep, and goats with 2 different types of tonometers. Can J Vet Res. 2018;82(3):208-15.

22. Gelatt KN, MacKay EO. Distribution of intraocular pressure in dogs. Vet Ophthalmol. 1998;1 (2-3):109-14.

23. Burke $J A$, Potter $D E$. The ocular effects of xylazine in rabbits, cats, and monkeys. J Ocul Pharmacol. 1986;2(1):9-21.

24. Kovalcuka L, Birgele E, Bandere D, Williams DL. Comparison of the effects of topical and systemic atropine sulfate on intraocular pressure and pupil diameter in the normal canine eye. Vet Ophthalmol. 2015;18(1):43-9.

25. Ofri R, Horowitz I, Jacobson S, Kass PH. The effects of anesthesia and gender on intraocular pressure in lions (Panthera leo). J Zoo Wild Med. 1998;29(3): 307-10.

26. Wu SY, Nemesure B, Hennis A, Leske MC, Barbados Eye Studies G. Nine-year changes in intraocular pressure: the Barbados Eye Studies. Arch Ophthalmol. 2006;124(11):1631-6.

\section{Publisher's Note}

Springer Nature remains neutral with regard to jurisdictional claims in published maps and institutional affiliations. 